\title{
Subjetividad y medios de vida sostenibles de hogares vulnerados por un desastre hidrometereológico en la región de Atacama de Chile
}

\author{
Subjectivity and sustainable livelihood in households affected by \\ hydrometeorological disasters in the Atacama region from Chile
}

\section{Subjetividade e meios de subsistência sustentáveis em famílias afetadas por um desastre hidrometeorológico na região do Atacama no Chile}

\author{
José Sandoval-Díaz ${ }^{1}$, ORCID 0000-0001-7247-7113 \\ Vesna Karmelic-Pavlov 2, ORCID 0000-0002-5015-1515 \\ Susy Tello-Cabrera ${ }^{3}$, ORCID 0000-0002-3291-3483 \\ Marjorie Chaparro-Guzmán ${ }^{4}$, ORCID 0000-0003-1056-7193 \\ Gianina Gaete-Bastias ${ }^{5}$, ORCID 0000-0001-6980-4689 \\ Karina Alfaro-Saldivar ${ }^{6}$, ORCID 0000-0002-1024-7475 \\ ${ }^{1}$ Departamento de Ciencias Sociales, Universidad del Bío-Bío. Chile \\ 23456 Escuela de Psicología y Filosofía, Universidad de Tarapacá. Chile
}

\begin{abstract}
Resumen: El incremento de riesgos hidrometereológicos, a causa del cambio ambiental global, ha intensificado la susceptibilidad de los medios de vida de hogares expuestos, conllevando la necesidad de fortalecer las capacidades de agencia y empoderamiento local. En función de esto, el presente trabajo analiza la dimensión subjetiva de las capacidades y medios de vida de hogares vulnerados por un desastre hidrometeorológico en Copiapó, Chile. Para esto, se utilizó un diseño de caso cualitativo seleccionando 15 jefe/as de hogar vulnerados por el desastre. Los datos son producidos mediante la entrevista episódica, utilizando como estrategia de análisis la teoría fundamentada. Los resultados obtenidos resaltan las (i) disposiciones tácticas de afrontamiento y (ii) la articulación entre capitales y estructura de oportunidades, en términos de medios de vida. Concluimos con el relevo de los aspectos psicosociales de la vulnerabilidad-capacidad ante desastres, en tanto factores mediadores entre el agenciamiento y las estructuras comunitarias, estatales y mercantiles.
\end{abstract}

Palabras claves: subjetividad; medios de vida sostenibles; capacidades de afrontamiento; vulnerabilidad social; desastre hidrometereológico

Abstract: The increase of the hydrometeorological risks due the environmental global change has intensified the susceptibility of livelihood in exposed households, leading the need of strengthen agency capacities and local empowerment. According to this, the investigation analyzes a subjective dimension of capacities and livelihoods of households affected by hydrometeorological disaster occurred in Copiapo city from Chile. For this, a qualitative case design was used, and 15 household heads affected by the disaster were selected. The data is produced through an episodic interview, using grounded theory as analysis strategy. The results obtained highlights the (i) tactical coping provisions, and (ii) the articulation between capitals, and opportunities structure in terms of livelihood. We conclude emphasizing the psychosocial aspects of vulnerability-capacity to disasters, as mediating factors between agency and community, state and commercial structures.

Keywords: subjectivity; sustainable livelihood; coping provisions; social vulnerability; hydrometeorological disaster 
Resumo: O aumento dos riscos hidrometeorológicos, devido à mudança ambiental global, intensificou a suscetibilidade dos meios de subsistência das famílias expostas, levando à necessidade de fortalecer as capacidades de agenciamento e empoderamento local. Em função disso, o presente estudo analisa a dimensão subjetiva das capacidades e meios de subsistência das famílias afetadas por um desastre hidrometeorológico em Copiapó, Chile. Para isso, foi utilizado um desenho estudo de caso qualitativo, selecionando 15 chefes/as de família afetados pelo desastre. Os dados são produzidos por meio de entrevista episódica, utilizando como estratégia de análise a teoria fundamentada. Os resultados obtidos destacam (i) os arranjos táticos de enfrentamento e (ii) a articulação entre capitais e estrutura de oportunidades, em termos de meios de subsistência. Concluímos com o repasso dos aspectos psicossociais da vulnerabilidadecapacidade frente a desastres, como fatores mediadores entre agenciamento e as estruturas comunitárias, estatais e mercantis.

Palavras-chave: subjetividade; meios de subsistência sustentáveis; capacidades de enfrentamento; vulnerabilidade social; desastre hidrometeorológico

Cómo citar:

Sandoval-Díaz, J., Karmelic-Pavlov, V., Tello-Cabrera, S., Chaparro-Guzmán, M., Gaete-Bastías, G., \& Alfaro-Saldívar, K. (2020). Subjetividad y medios de vida sostenibles de hogares vulnerados por un desastre hidrometereológico en la región de Atacama de Chile. Ciencias Psicológicas, 14(2), e-2287. doi: https://doi.org/10.22235/cp.v14i2.2287

Correspondencia: José Sandoval-Díaz: Centro de Estudios Ñuble, Grupo de Investigación en Ciudadanía y Equidad y Departamento de ciencias sociales de la Universidad del Bío-Bío, Chillan, Chile. Andrés Bello 720 , Chillán, Chile. E-mail: jsandoval@ubiobio.cl. Vesna Karmelic-Pavlov: Escuela de Psicología y Filosofía de la Universidad de Tarapacá, Arica, Chile. Universidad de Tarapacá 18 de septiembre 2222, Arica, Chile. E-mail: vkarmeli@academicos.uta.cl. Susy Tello-Cabrera,e-mail: tello.susy@gmail.com. Marjorie Chaparro-Guzmán,email: marjorie0601@gmail.com. Gianina Gaete-Bastías, e-mail: gianninagaete@hotmail.com. Karina AlfaroSaldivar, e-mail: karina.alfaro.saldivar@hotmail.com

\section{Introducción}

Las estrategias de mitigación y adaptación ante el cambio ambiental global se han posicionado fuertemente en las agendas políticas-institucionales regionales, nacionales y locales (Günther \& Gutiérrez, 2017; Intergovernmental Panel on Climate Change [IPCC], 2014). En términos de impacto global, sólo considerando el año 2018, los desastres climatológicos vulneraron a un total de 57,3 millones de personas, siendo liderado por las inundaciones, con un promedio de 35.4 millones de afectados (UN Office for Disaster Risk Reduction [UNDRR], 2019).

En América Latina (AL), esta problemática socioambiental no sólo ha conllevado la emergencia de nuevos riesgos, tales como él (i) aumento del nivel del mar, (ii) olas de calor, (iii) pérdida de biodiversidad e (iv) intensificación de peligros extremos hidrometeorológicos; Sino que, a su vez, ha profundizado e intensificado los riesgos modernos inmanentes a la región, tales como el empobrecimiento, desigualdad y vulnerabilidad socioestructural (Sandoval-Díaz, 2020; Wisner, Blaikie, Cannon \& Davis, 2004). 
Estos intensivos/extensivos riesgos socioambientales, han conllevado la reconfiguración de las estrategias de gobernanza de reducción de riesgo desastre (RRD), trasladando el foco desde los gobiernos centrales, hacia el fortalecimiento multisectorial de la resiliencia y capacidades a escala territorial-local (Sandoval \& Sarmiento, 2018; UNDRR, 2015). Este desarrollo y fortalecimiento de capacidades locales incluiría no sólo las dimensiones (i) físicomateriales, (ii) social-organizacionales y (iii) disposicional-motivacionales (Anderson \& Woodrow, 1989, Burkett, 2013; Sandoval et al. 2018), sino también a la flexibilidad adaptativa del saber practico, del cuándo, dónde y cómo, desplegar esta configuración de capacidades (Gaillard, Cadag \& Rampengan, 2019; Kendra, Clay \& Gill, 2018; Rojas \& Sandoval, 2020).

A escala local, tanto barrios $y$ hogares presentan grados diferenciados de vulnerabilidades-capacidades ante el proceso de riesgo de desastre (Berroeta \& Pinto de Carvalho, 2020; Sandoval Díaz \& Astudillo Pizarro, 2019). Para los hogares, el despliegue de capacidades de afrontamiento dependerá tanto de las practicas sociales de acceso a recursos simbólicos/materiales (y su movilización en forma de activos), como de su articulación a las estructuras de oportunidades multiescalares, sean el Estado, Mercado y/o la Comunidad (Arteaga \& Pérez, 2011; Rojas \& Sandoval, 2020). Esta articulación dialéctica entre la estructura y el agenciamiento de los hogares, configuran modos y medios de vida, que pueden verse debilitados o fortalecidos ante una situación de desastre (Marín, Bedoya \& Cárdenas, 2015; Räsänen et al. 2016; Rakodi \& Lloyd-Jones, 2014).

De acuerdo con Serrat (2017), el marco de medios de vida (ver Figura 1) permitiría explorar la interacción entre las capacidades de afrontamiento de hogares (en término de capitales $^{1}$ ) y el contexto de vulnerabilidad social ante una situación de crisis, como lo son los desastres socionaturales.

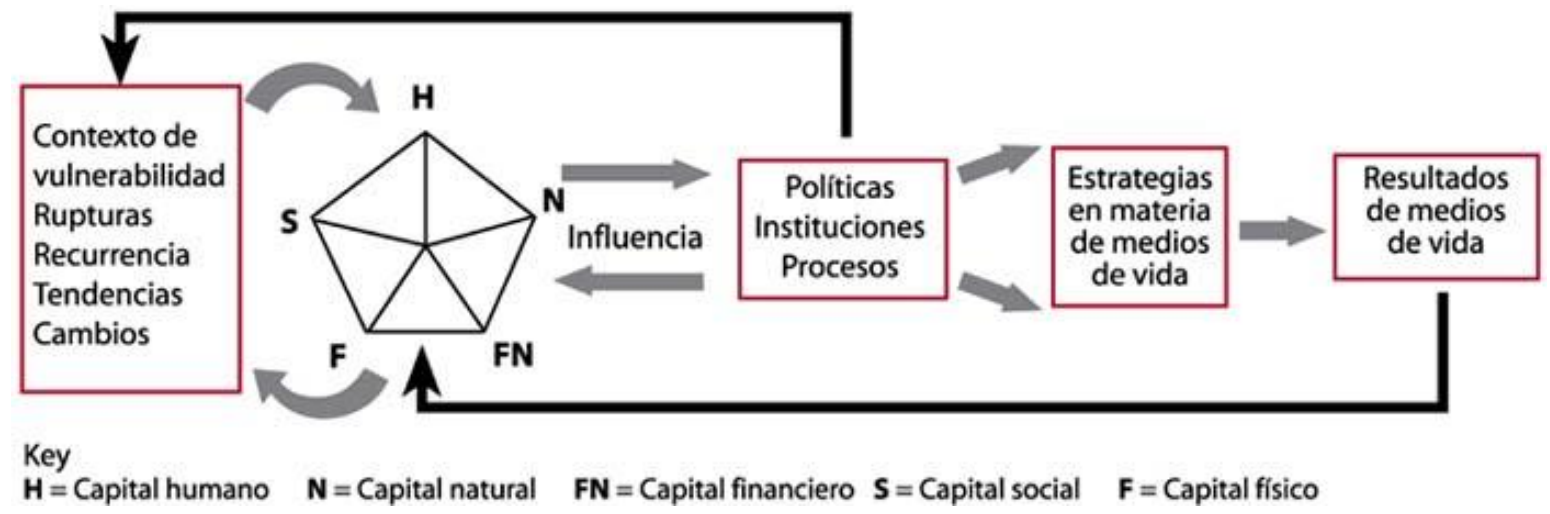

Figura 1.

Marco de los medios de vida sostenible (MVS). Fuente: Organización de las Naciones Unidas para la Alimentación y la Agricultura [FAO] (s.f).

\footnotetext{
${ }^{1}$ De acuerdo con Bourdieu (2014) entendemos capital como el conjunto de activos (que integra, pero desborda los recursos económicos-financieros), el cual permite a los agentes participar en un determinado campo, en tanto activos informacionales (capital cultural), de adscripción a grupos (capital social) o socioculturales de orden simbólico (capital simbólico).
} 
Bajo este marco, se identifican tres componentes centrales:

(i) El contexto de vulnerabilidad: Vinculado a procesos de producción de riesgo socionaturales y/o antrópicos, tornando susceptibles los medios de vida de hogares y comunidades expuestas a estos riesgos.

(ii) Capitales configuradores de MVS: Referidos a la base movilizada de cinco activos:

(a) Humano: nivel de salud, alimentación y educación, (b) Social: redes entre individuos con intereses compartidos, formas de participación social y confianza, (c) Natural: recursos ambientales, (d) Físico: infraestructuras/equipamientos para las necesidades básicas y (re)productivas, y (e) Financieros: recursos monetarios.

(iii) Las estructuras de oportunidades: Procesos externos globales, regionales, nacionales y/o locales que influyen en la configuración de MVS, sea a nivel de Estado, Mercado y/o la Comunidad. Estas instituciones incluyen organizaciones formales, informales, políticas y prácticas socioculturales, así como también, las invisibles "reglas del juego" normativas societales.

En síntesis, una experiencia de afrontamiento adaptativa se vinculará al acceso y movilización interdependiente de capitales a nivel de hogar, logrando la sostenibilidad de los medios de vida (Marín et al. 2015; Scoones, 2009). A su vez, una estructura política e institucional que ofrezcan oportunidades de Desarrollo facilitará a los hogares la consecución de acceso a recursos/activos necesarios el fortalecimiento adaptativo de estos (Rakodi \& LloydJones, 2014); Mientras que factores externos, tales como condiciones de aislamiento, daño severo y estructuras societales obstaculizadoras, intensificaran la susceptibilidad de las comunidades con menor agencia ante el desastre (Marín, Bodin, Gelcich \& Crona, 2015).

No obstante, bajo este afrontamiento agentivo de los hogares, subyacen componentes subjetivos mediadores ante la vulnerabilidad social, los cuales posibilitan o inhabilitan el despliegue de capacidades de afrontamiento tácticas-resistentes y/o estratégicas-resilientes (Arteaga \& Pérez, 2011; Sandoval, 2017; Sandoval Díaz \& Astudillo Pizarro, 2019). En consecuencia, esta dimensión subjetiva emerge bajo una configuración dialéctica entre sentidos y afectos, gatilladas bajo la experiencia materializada (colectiva-relacional y sentida-personal) del proceso de riesgo de desastre; Mediando así entre el despliegue de prácticas y/o capacidades de afrontamiento y la situación de crisis (Arteaga et al. 2015; Rojas \& Sandoval, 2020).

A partir de estos antecedentes, el presente trabajo tiene como objetivos: (i) caracterizar la dimensión subjetiva de las capacidades de afrontamiento de hogares vulnerados por un desastre hidrometereológico y (ii) analizar la articulación entre los capitales y las estructuras de oportunidades de los medios de vida de los hogares ante el desastre.

\section{Características del caso de estudio: desastre hidrometereológico del 25 de marzo del 2015 (25M)}

Un evento hidrometeorológico extremo, producto de una baja segregada asociada a precipitaciones, causó lluvias torrenciales en 17 quebradas de la región de Atacama, Chile. En la ciudad de Copiapó, estas inundaciones arrastraron gran cantidad de agua, material particulado y relaves mineros, enterrando la ciudad bajo una capa de sedimentos de $31 \mathrm{~cm}$ de espesor. En cuanto a su impacto, se registraron 22 fallecidos, 28.000 damnificados, 2.000 viviendas destruidas y 5.000 con daño mayor, sumando un impacto económico superior a los 46 millones de dólares (Astudillo Pizarro \& Sandoval Díaz, 2019). En tanto al impacto psicosocial, los grupos expuestos-susceptibles que presentaron una significativa severidad subjetiva de daño, tras el desastre, fueron los adulto/as mayores, personas con discapacidad/enfermedad crónica, de sexo femenino, con bajos ingresos económicos y sin educación superior, sumándose quienes se encuentran en la condición de daño parcial-total en su vivienda y no recibieron apoyo para la reconstrucción habitacional (Sandoval-Díaz \& Cuadra-Martínez, 2020). 


\section{Metodología}

Se utilizó un diseño de estudio de caso cualitativo, definido como el examen profundo y contextualizado de diversos aspectos psicosociales del desastre, tales como los significados, prácticas y materialidades emergentes, esto desde la perspectiva fenomenológica de los jefes/as de hogar (Coller, 2005; Flick, 2007).

\section{Participantes y procedimientos}

Por medio de un muestreo teórico acumulativo (Carrero, Soriano \& Trinidad, 2012), se entrevistaron a 15 jefe/as de hogar, quienes cumplieron los siguientes criterios de inclusión muestral: i) daño severo y/o pérdida total de la vivienda por el desastre, ii) residencia mínima de un año en la región, iii) hogares compuesto por un mínimo de dos integrantes. En términos metodológicos entendemos hogar "un grupo de personas que residen conjuntamente, las cuales contribuyen y/o se benefician de una economía conjunta o trabajo doméstico" (Rakodi \& LloydJones, 2014 p.7).

En términos procedimentales, las entrevistas fueron realizadas por el primer autor a los participantes en sus respectivas viviendas o en el albergue de emergencia posdesastre. El levantamiento de datos se realizó en las dos primeras semanas de abril del año 2015 (a dos semanas del $25 \mathrm{M}$ ), presentando una duración promedio entre 45-60 minutos, previa lectura y firma del consentimiento informado.

\section{Estrategia de producción y análisis de información}

Se utilizó la entrevista episódica, permitiendo profundizar en la experiencia, significados y practicas ante el proceso de desastre (Flick, 2007). Se elaboró una pauta temática en base a dos criterios organizativos:

a) Temporalidad: Centrada en el proceso de riesgo-desastre, específicamente, en las etapas de respuesta, emergencia y rehabilitación temprana.

b) Capacidades de afrontamiento y estructura de oportunidades: Centrada en las disposiciones, sentidos y afectos que mediaron la articulación entre capitales y las estructuras de oportunidades.

Posterior a la transcripción íntegra de las entrevistas, se utilizó el proceso de codificación straussiano de la teoría fundamentada "orientada a un trabajo de investigación más enraizado en la descripción interpretativa que en la construcción de teoría formal emergente" (Carrero et al. 2012, 19). En términos procedimentales, el proceso de codificación se dividió en dos etapas: i) abierta y ii) axial, utilizando como herramienta de apoyo el software Atlas Ti v7.

\section{Resultados}

Los resultados se dividen en dos tópicos: 1) mediaciones subjetivas de las capacidades de afrontamiento y 2) configuración de medios de vida. Finalmente, cada resultado es acompañado de un esquema de la codificación axial y citas textuales anonimizadas, identificando algunos marcadores de posición, tales como sexo y edad.

\section{Mediaciones subjetivas de la vulnerabilidad social}

En el esquema de la figura 2, la categoría central son las mediaciones subjetivas de la vulnerabilidad social. De esta red de afectos y sentidos emergen tres tipos de capacidades de afrontamiento tácticas-resistentes: i) solidaridad, ii) autogestión familiar y iii) resignación. 


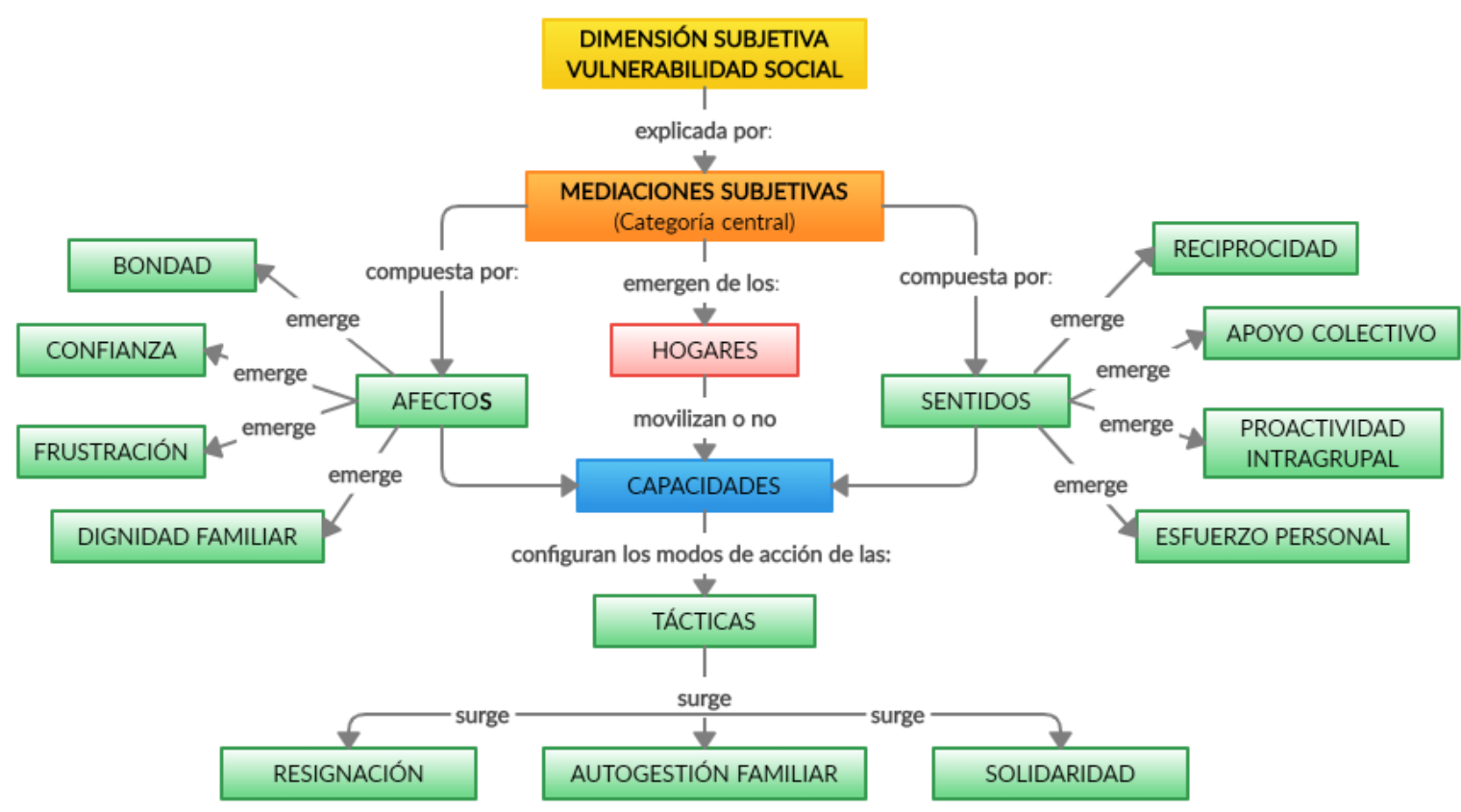

Figura 2.

Mediaciones subjetivas de la vulnerabilidad social. Fuente: Elaborado por Saron Monsalves en Atlas ti.7.

1.1 Táctica de solidaridad: Capacidad desplegada para hacer frente la emergencia, esto bajo los sentidos subjetivos de "reciprocidad y apoyo colectivo". Ambos sentidos, movilizan el recurso latente del "capital social territorial" de la estructura de oportunidad Comunitaria. Así mismo, esta táctica integra los afectos temporales de "bondad y confianza", los cuales son significados como un bálsamo amortiguador ante la angustia que depara la incerteza de la etapa de reconstrucción.

Todos los insumos que tenía los doné acá el primer día, cuando volví al local había pollos, papas, saqué y los doné. Hicimos un asado, yo creo que fue el único albergue que hizo asado, fue muy cómico, entonces empezó a llegar todo el mundo por el olor rico que salía... de alguna forma fue como un bálsamo para nuestra angustia, porque saber que ellos por lo menos estaban disfrutando de un momento rico (Hombre, 40 años).

La solidaridad que ha mostrado la gente de acá, los vecinos que yo ni conocía que con suerte los miraba de lejos. Entonces la solidaridad que han mostrado funcionó, sirvió como para animarme un poco (Mujer, 25 años).

1.2 Táctica de autogestión: Capacidad desplegada con miras a la sobrevivencia del día a día, esto bajo los sentidos de "reciprocidad y proactividad intragrupal". A su vez, esta táctica se encuentra cruzada por las prácticas del "apoyo de terceros", utilizando, así como principal estructura de oportunidad tanto el Mercado (almacenes de barrio) y/o el Estado central, pese a la burocracia percibida hacia este último. El afecto que prima es la "frustración", esto ante la intensificación de la vulnerabilidad social (gatillada tras el desastre y profundizada por los organismos gubernamentales encargados de su gestión), siendo pobremente mitigada por el "esfuerzo personal" de los jefe/as de hogar.

Lamentablemente las reacciones de ayuda de parte del gobierno son muy lentas, anoche me trajeron un formulario para que lo llenara con los daños, y que la 
fuera a dejar a la oficina comunitaria ... mire yo perdí todo, pero como sea voy a arreglar, voy a levantarme sólo para echar andar el negocio (Mujer, 60 años). El sistema es lento y de hecho hasta hoy día recién escuché por primera vez que llegó alguna ayuda del gobierno, creo que un paquete de productos de aseo. Antes de eso toda la mercadería que ha estado acá fue entregada por los chiquillos voluntarios y la he tenido que pedir fiado en el almacén del vecino (Hombre, 33 años).

1.3 Táctica de resignación: Capacidad desplegada bajo el sentido de "esfuerzo personal", con miras a la recuperación del estilo de vida arrebatado por el desastre. Así mismo, esta táctica es mediada por el afecto de la "dignidad familiar", la cual genera que los hogares soslayen el apoyo de terceros, sean Estatales o de la Comunidad, esto para no ser percibidos como "limosneros de subsidios gubernamentales" (pordiosear), como por la injusticia percibida hacia los "pitutos" (contacto útil) que terceros utilizan "ilegalmente" para acceder a beneficios. Estos elementos resultan claves para comprender la importancia de la mantención, a como dé lugar, del estatus social, vía endeudamiento crediticio a través del Mercado financiero.

A mí me carga andar metida en la municipalidad porque es como que tienes que andar limosneando ... aquí las amigas de las amigas pueden sacar cosas ...o sea los que tienen pitutos por eso te digo, que los primeros días no tenía que darle de comer a mis hijos ... le daba fideos solos y eso... (Mujer, 45 años).

No me quedó otra que pedir prestado, pedir "fiado" (pagar más adelante) en el almacén. Ahora he tenido que utilizar las tarjetas de las tiendas para comprar las cosas que se perdieron (electrodomésticos) ... usted sabe la vida tiene que seguir y no nos queda otra que seguir trabajando (Mujer, 35 años).

\section{Configuración de MVS}

La Figura 3 da cuenta del acceso y movilización de capitales en las tres estructuras de oportunidades centrales: Estado, Mercado y Comunidad. Su despliegue, sin embargo, se encuentra condicionado a cuatro barreras de la vulnerabilidad socioestructural: a) exposición geográfica multiamenaza, b) desorganización institucional c) infraestructura habitacional precaria y d) pérdida de fuente laboral. En algunos casos, dichos obstáculos agudizaron la perdida e insostenibilidad de los MVS, incrementando aún más las trayectorias vulnerabilizadas de los hogares tras el desastre. 


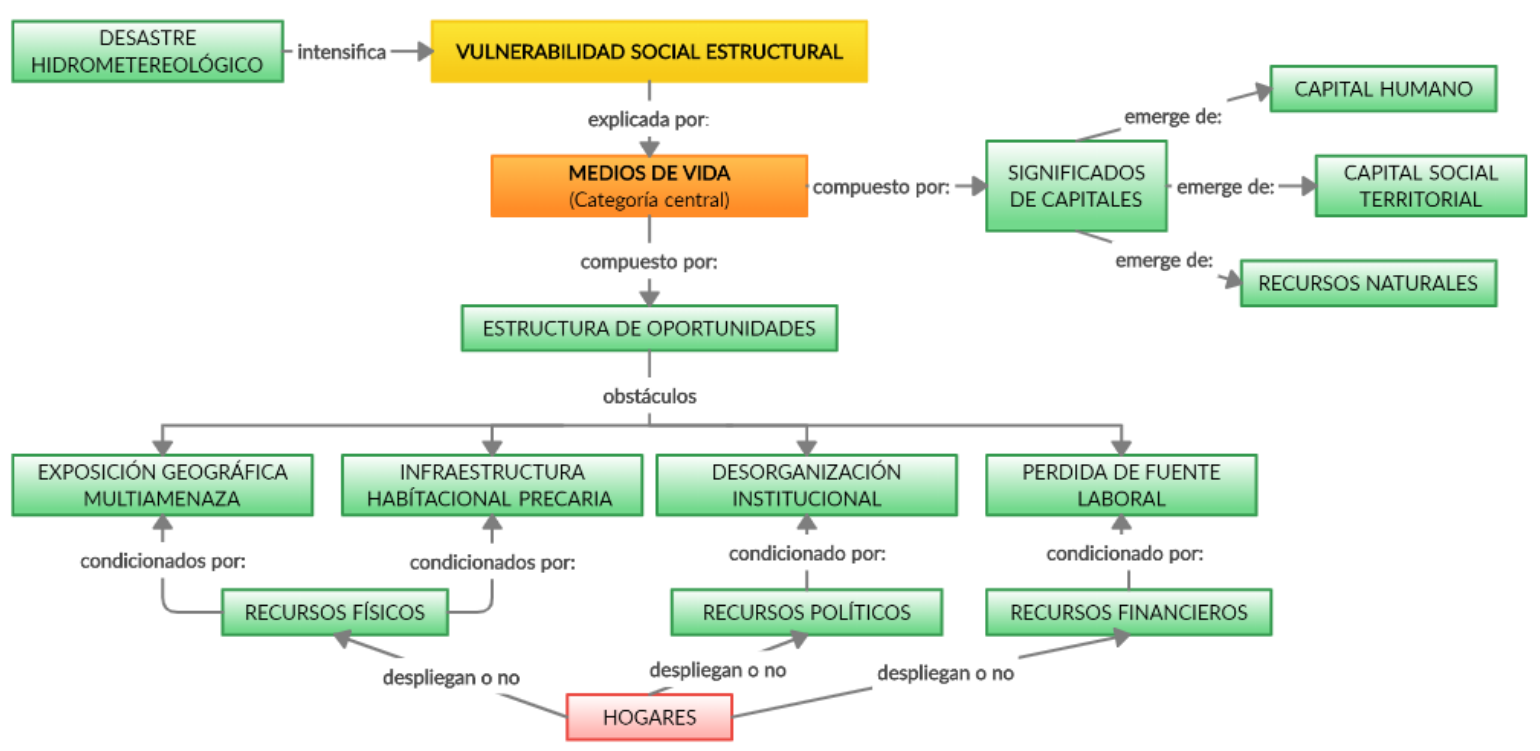

Figura 3.

Configuración de Medios de Vida Sostenibles de los hogares. Fuente: Elaborado por Saron Monsalves en Atlas ti.7.

\subsection{Obstáculos de las estructuras de oportunidades}

2.1.1 Exposición geográfica multiamenaza: Previo al 25M, Atacama se caracteriza por ser un territorio extractivo de recursos naturales, lo cual se ha intensificado con la apertura de la inversión transnacional/estatal de la minería y agroindustria a gran escala (Sandoval-Díaz, 2020). De acuerdo con la causa Relaves (s.f), Atacama es la segunda región con mayor cantidad de relaves ${ }^{2}$ a nivel nacional, de los cuales 30 se encuentran activos, 108 inactivos y 23 abandonados. Esto constituye una fuente de riesgo ambiental para la salud poblacional, lo cual se materializo en un desastre antrópico tras las inundaciones del $25 \mathrm{M}$, esto al movilizar elevadas concentraciones de metales pesados, sumándose también la cantidad de material particulado activa por la acción eólica (Cortés \& Tchernitchin, 2018).

Por otro lado, debido a una ausencia histórica de una política nacional de ordenamiento territorial, algunas viviendas (recurso físico) colindan con el lecho del cauce del rio, presentando una mayor exposición-susceptibilidad ante potenciales inundaciones.

Yo crecí con la contaminación minera... también tenemos el polvo en suspensión que es pan de todos los días por las tronaduras mineras (Mujer, 25 años).

Lamentablemente yo creo que soy uno de los más afectado de esta calle, tengo un terreno gigante que está conectado con el rio, entonces cuando se vino esto por ambos lados, quedé encerrado en mi propiedad, lo cual fue un daño mucho más grave... entonces mi local se inundó completo (Hombre, 40 años).

2.1.2 Burocracia y desorganización institucional: El segundo obstáculo se vincula a la desorganización gubernamental (recurso político) a lo largo de la gestión de la emergencia, bajo la cual opero los mismos protocolos burocráticos utilizados en "situaciones de no excepcionalidad" (Rojas \& Sandoval, 2020). Respecto a esto, a nivel nacional las políticas de recuperación posdesastre han tendido a ser reducidas a su dimensión física-material, esto bajo los términos de rehabilitación de la infraestructura de conectividad dañada, como por la reconstrucción habitacional "viviendista" (Contreras \& Beltrán, 2015), comprendiendo a los

\footnotetext{
${ }^{2}$ Conjunto de desechos de procesos mineros de la concentración de minerales, usualmente constituido por una mezcla de rocas molidas, agua y minerales.
} 
hogares como meros receptores de subsidios habitacionales, fracturado así el capital social comunitario subyacente a los lugares afectados (González-Muzzio, 2013).

Los voluntarios y vecinos, no hay nada que hablar muy solidarios, pero las instituciones de gobierno muy lentas, no tengo para cuando recibir mi casa, sólo sé que tengo que volver al SERVIU ${ }^{3}$ a postular y hacer todo de nuevo (Hombre, 46 años).

Hay que hacer cola para todo, para el agua, para que te den un paquete de fideos y con cuatro niños y sin apoyo es difícil (Mujer, 45 años).

2.1.3 Infraestructura habitacional precaria: Atacama ocupa el primer lugar nacional en déficit habitacional (recurso físico), equivalente a 18.962 viviendas en hacinamiento medio-crítico y/o en condiciones de saneamiento deficitarias (Cámara Chilena de la Construcción [CChC], s.f). Este alto porcentaje se vincularían al aumento sostenido de familias que viven en condición de allegadas, correspondientes al 9\% de la población total (Moreno, 2019). Respecto al impacto del desastre, la ciudad quedó bajo una capa de sedimentos de $31 \mathrm{~cm}$ de espesor, llegando en algunos sectores a los dos metros de altura (ver Figura 4).

Aquí vivimos 12 personas, la familia de mi hermana, la mía y mis papas... estamos hacinados como ratones desde que ocurrió todo esto (Mujer, 45 años).

El agua estaba hasta acá (indica altura en la pared) y el barro hasta por ahí, ahí están las marcas ve... lo que más me interesaba para ocupar era el baño y la ducha ...Pero no se puede ocupar porque está tapado ... Se metió una maquina chica allá y rompió las cañerías las tuberías... esta la pura embarrada (Hombre, 38 años).

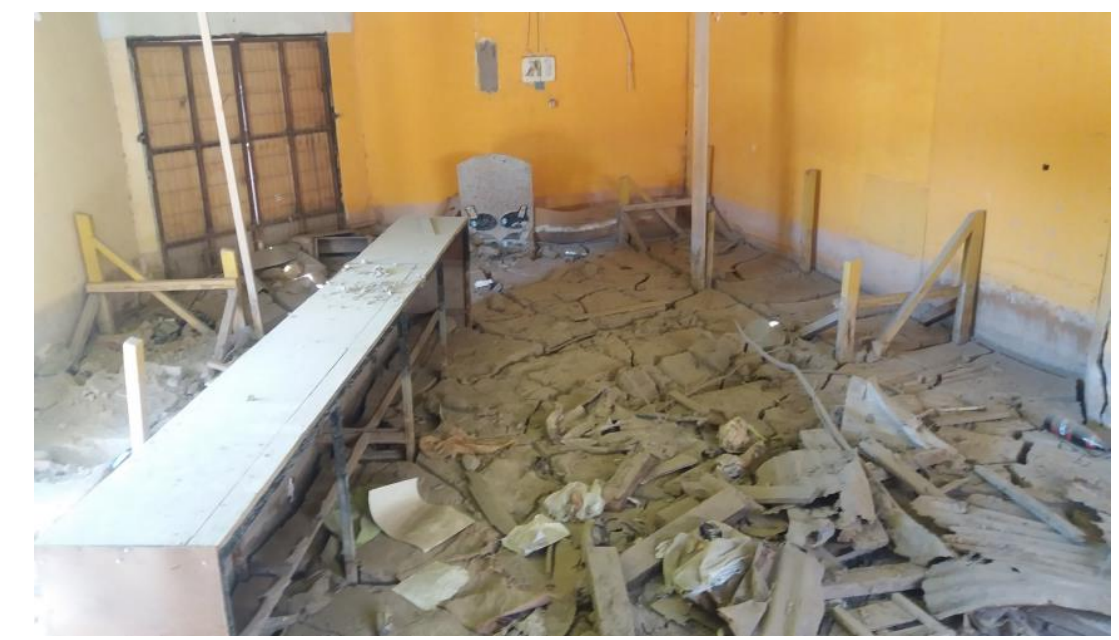

Figura 4.

Vivienda abandonada posdesastre hidrometereológico. Fuente: Fotografia tomada por José Sandoval.

2.1.4 Pérdida de fuente laboral: Esta dimensión es relevante para la reproducción del recurso financiero de los MVS de los hogares, presentando distintos grados de afectación. Quienes resultaron mayormente afectados son aquellos hogares sujetos al autoempleo, cuya fuente laboral utilizan su vivienda como activo, bajo la fuerza de trabajo familiar (ej. almacenes de barrio). En segundo lugar, si bien las familias asalariadas no vieron mermados sus ingresos, si se vieron conflictuados entre el ocuparse de las necesidades inmediatas de rehabilitación de la vivienda, las dinámicas de organización familiar y el tener que retornar al trabajo.

\footnotetext{
${ }^{3}$ Servicio de Vivienda y Urbanización del gobierno de Chile.
} 
Mi local se inundó hasta sobre la rodilla, casi llegando a la cintura y toda la maquinaria se quedó abajo. Hasta el día de hoy estamos limpiando con la esperanza que se salve alguna maquinaria ... pero como son cosas electrodomésticas es muy difícil, porque estuvieron bajo el agua con barro varios días (Hombre, 40 años).

Es difícil volver a la normalidad cuando en la casa está la escoba (desorden), pero bueno, uno tiene que volver al trabajo, porque al final es la única forma de seguir saliendo para delante (Hombre, 38 años).

\subsection{Acceso y movilización de capitales}

Los hogares entrevistados poseen tipos y grados diferenciados de capital, configurando así distintas opciones de MVS; Sin embargo, la situación de vulneración tras el desastre, y las barreras obstaculizadoras de la estructura de oportunidades emergentes, limitaron su acceso y movilización.

En primer lugar, en cuanto al capital humano, los hogares significan la salud física como un impedimento preexistente, ejemplificado tanto por los antecedentes familiares de enfermedades crónicas, como por aquellas vinculadas a la adultez mayor, acrecentándose aún más tras el desastre. Sumado a esto, las etapas de respuesta-emergencia demandaron una alta autogestión familiar, la cual se vio traducida en un marcado desgaste físico y psicológico, en términos de severidad subjetiva (Sandoval-Díaz \& Cuadra-Martínez, 2020).

En términos de recursos físicos colectivos, el centro de salud comunitario (y otros servicios de salud públicos/privados), se vieron inhabilitados por las inundaciones, postergando el acceso de quienes esperaban atención o se encontraban en tratamiento previo.

Yo tengo artrosis en esta rodilla y donde estaba sentada en la camioneta era un dolor constante en mi pierna (Mujer, 66 años).

No puedo carretillar (acción de usar carretilla) mucho porque tengo quebrado este brazo, mi hermano es enfermo epiléptico y mi madre tiene mucha edad ya ... la verdad todo esto me tiene muy agotado (Hombre, 38 años).

Llevamos trece días tomando "medicamentos", estamos los dos enfermos, resfriados, con gripe, ahora recién ella empezó a sanarse yo hasta el día de hoy estuve un poco más ... pero no podemos hacer nada tampoco por que el CESFAM ${ }^{4}$ está lleno de barro (Hombre, 40 años).

En segundo lugar, se destaca el papel central del capital social territorial, significado como aquellas acciones organizadas en base a normas y redes, que logran movilizar recursos y respuestas colectivas ante la emergencia (Adger \& Brown, 2009). Para el presente caso, esto se comprende bajo el contexto de apoyo social familiar, tanto físico-material como simbólicoemocional, involucrando distintas acciones tales como: (i) salvaguardar la vida de sus integrantes, (ii) soporte emocional continúo y (iii) autogestión y (iv) empoderamiento familiar. En esta línea se ha encontrado que el comportamiento prosocial, el empoderamiento y el sentido de pertenencia comunitario, pueden ayudar a reducir efectos psicosociales negativos de los desastres (Alvarado, Pradenas, Yáñez, Cuadra \& Sandoval, 2019).

No quería moverse de ahí... me costó convencerla, yo le decía si estamos los dos todo estará bien, esto lo podemos levantar de "nuevo" y me costó, pero la convencí (Hombre, 55 años).

En tercer lugar, dada las características paisajísticas de la comuna, emerge la importancia atribuida a distintos recursos naturales. A escala territorial se menciona la escasez de espacios verdes para la recreación comunitaria; Mientras que, a nivel familiar, se significa la "pérdida de animales" bajo dos sentidos: el primero vinculado los lazos afectivos de mascotas perdidas tras

\footnotetext{
${ }^{4}$ Centros de Salud Familiar vinculados a la atención primaria publica de Chile.
} 
el desastre; y el segundo vinculado a la producción alimentaria de pequeña escala (ej. gallinas ponedoras). De acuerdo con esto último, el Marco de Sendai para la RRD no sólo se enfoca en salvar vidas y proteger la infraestructura física, sino también, incluye la protección de activos productivos, incluyendo ganado, animales de trabajo, herramientas y semillas (UNDRR, 2015).

Tenía unas gallinas en un gallinero, que está ahí, las pobrecitas quedaron pegadas en el barro, no podían salir así que mi hijo me las sacó de ahí, me las metió en una bodega, todavía las tengo en una bodega allá, una se murió y la otra se va a morir parece porque no puede caminar con el hielo en las patitas, no puede caminar, así que se salvaron dos no más, esas dos que están ahí [apuntando el lugar] (Mujer, 66 años).

\section{Discusión y Conclusiones}

El presente artículo tuvo como objetivo analizar la dimensión subjetiva de la vulnerabilidad social y de los MVS de hogares afectados por un desastre hidrometereológico. En primer lugar, los hogares desplegaron las capacidades tácticas-resistentes de i) solidaridad, ii) autogestión y iii) resignación, relevando la existencia de racionalidades heterogéneas y en ocasiones conflictivas, las cuales propician disposiciones agentivas de acción diferenciadas (Lillo, Prosser, Oróstica, \& Pérez, 2018). Este rol activo de los afectos y sentidos subjetivos, si bien no logran romper con el contexto de vulnerabilidad socioestructural, posibilitan condiciones empoderadoras disposicionales de afrontamiento resistentes ante el proceso de riesgo-desastre (Rojas-Páez \& Sandoval-Díaz, 2020; Sandoval, 2017). No obstante, esta excesiva sobrecompensación agencial contrarresta con la incapacidad articuladora de las estructuras institucionales vinculadas a la RRD, dificultando la adquisición y/o desarrollo de capacidades adaptativas-resilientes locales (Sandoval-Díaz, 2020).

En segundo lugar, si bien los hogares poseen y acceden a los capitales i) humano, ii) físico y iii) financiero, su despliegue se vio fragilizado tanto por la intensificación de la vulnerabilidad tras el desastre, como por las obstáculos estructurales-institucionales, dando cuenta del efecto multiplicador negativo que presentaron las condiciones de (i) exposición geográfica multiamenaza, (ii) infraestructura habitacional precaria, (iii) pérdida de fuente laboral y (iv) desorganización institucional (Marín et al. 2015; Rakodi \& Lloyd-Jones, 2014). Sin embargo, esta emersión de trayectorias de vulnerabilidad fue resistida vía despliegue del capital social territorial (González-Muzzio, 2013) y el Mercado crediticio vía endeudamiento (Arteaga \& Pérez, 2011); Y en menor medida con recursos del Estado, dado su predominante rol subsidiario (Arteaga et al. 2015). En términos institucionales se señala la ausencia de un sistema integrado de gestión de RRD (Ugarte \& Salgado, 2014) y la (sobre)utilización de mecanismos rígidos tradicionales ante situaciones de excepcionalidad, como lo fue la reconstrucción habitacional (Contreras \& Beltrán, 2015).

Por tanto, estas trayectorias de vulnerabilidad se caracterizaron no sólo por la incapacidad de acceder y movilizar capitales, sino también por la falta de elección de estrategias alternativas de MVS, siendo los hogares más empobrecidos quienes adoptaron tácticas de sobrevivencia, por sobre estrategias adaptativas de Desarrollo y/o resiliencia (Marín et al., 2015; Scoones, 2009).

Respecto a los medios de vida configurados, la mayoría de los hogares significaron el i) recurso físico bajo el sentido de infraestructura pública y habitacional expuesta al riesgo, los ii) recursos financieros como medida de sustento de la reproducción familiar, el iii) capital humano vinculado al acceso a salud, mientras que el iv) capital social se asoció al apoyo y cohesión familiar-vecinal, y por último, los v) recursos naturales se vincularon a necesidad de espacios públicos verdes y la tenencia de animales. La escasez de vi) recursos políticos se vinculó tanto a la baja capacidad de procesamiento que presentó la estructura gubernamental ante lo ocurrido, así como también, a la ausencia de organizaciones colectivas de la sociedad civil que canalizaran 
las distintas demandas posdesastre (Astudillo Pizarro \& Sandoval Díaz, 2019; Contreras \& Beltrán, 2015; Ugarte \& Salgado, 2014).

En términos prospectivos, estos diferentes tipos de capital (y sus potenciales configuraciones en MVS), son relevantes no sólo para la adaptación ante el cambio ambiental global en curso, y los eventos extremos que este intensifica (Mabuku, Senzanje, Mudhara, Jewitt, \& Mulwafu, 2019), sino también para el fortalecimiento de una gobernanza del riesgo sustentada en la escala local-territorial (Sandoval \& Sarmiento, 2018).

Por último, a nivel práctico se desprenden las siguientes orientaciones programáticaspolíticas:

a) Revitalizar las perspectivas de los MVS ante los nuevos y dinámicos desafíos civilizatorios, requiriendo primero, un reconocimiento básico del papel del cambio ambiental global sobre la relación sociedad-naturaleza (Günther \& Gutiérrez, 2017); y, en segundo lugar, enriquecer la perspectiva estructural-agencial del enfoque, incorporando los componentes de producción de conocimiento, poder, valores y de cambio político (Sandoval-Díaz, 2020; Scoones, 2009).

(b) Tener en cuenta los diferentes factores que influyen en la elección de tácticas/estrategias de MVS, siendo el cambio climático sólo una más de las dimensiones estresantes que afectan la vida cotidiana de las comunidades expuestas (Räsänen et al. 2016). Para esto es relevante diseñar planes de RRD que incluyan las dimensiones psicosociales y subjetivas, esto bajo una perspectiva dialéctica-dialógica con el contexto de vulnerabilidad socioestructural (Berroeta \& Pinto de Carvalho, 2020).

(c) La necesidad de fortalecer las capacidades de afrontamiento para la RRD, comprendiéndose como (i) extensiones de la vida cotidiana y (ii) endógenas e idiosincráticas, es decir dependientes del contexto sociocultural (Gaillard et al. 2019); No obstante, esto debe ir acompañado de la reducción espacial de la vulnerabilidad socioestructural como medida de mediano-largo plazo (Astudillo Pizarro \& Sandoval Díaz, 2019; Sandoval et al. 2018).

(d) Otorgar una alta prioridad a la educación sobre el cambio climático, riesgos socioambientales y estrategias de adaptación, incorporando no sólo el conocimiento de expertos, sino también los saberes y memoria sociocultural de y desde las propias comunidades territorializadas (Mabuku et al. 2019; Marín et al. 2015).

\section{Referencias}

Adger, W. \& Brown, K. (2009). Vulnerability and resilience to environmental change: ecological and social perspectives. En N. Castree., D. Demeritt., D, Liverman \& B, Rhoads (Eds.) A companion to environmental geography, (pp. 109-122). Oxford: Blackwell Publishing Ltda. doi: 10.1002/9781444305722

Alvarado, R., Pradenas, C., Yañez, N., Cuadra, D. \& Sandoval, J. (2019). Teorías subjetivas del comportamiento prosocial: significados, desarrollo y motivaciones de jóvenes voluntarios ante un desastre socionatural. Liberabit, 25(2), 251-266. doi: 10.24265/liberabit.2019.v25n2.08

Anderson, M. \& Woodrow, P. (1989). Rising from the ashes: development strategies in times of disaster. Boulder: Westview Press

Arteaga, C. \& Pérez, S. (2011). Experiencias de vulnerabilidad: de las estrategias a las tácticas subjetivas. Universum (Talca), 26(2), 67-81. doi: 10.4067/S0718-23762011000200004

Arteaga, C., Pérez, S., Castro, F., Fava, D., Molina, G. \& Ramírez, C. (2015). Recursos, estructura de oportunidades y subjetividades en contextos de desastre. Análisis a partir del caso de Chaitén, En C. Arteaga \& R. Tapia (Eds.), Vulnerabilidades y desastres socionaturales (pp. 101-115). Santiago: Editorial Universitaria. 
Astudillo Pizarro, F. \& Sandoval Díaz, J. (2019). Justicia espacial, desastres socionaturales y políticas del espacio: dinámicas sociopolíticas frente a los aluviones y proceso de recuperación en Copiapó, Chile. Cuadernos de Geografía: Revista Colombiana de Geografía, 28(2), 303-321. doi: 10.15446/rcdg.v28n2.73520

Berroeta, H. \& Pinto de Carvalho, L. (2020). La Psicología Ambiental-Comunitaria en el Estudio de los Desastres: La Importancia de los Vínculos Socioespaciales. Psykhe, 29(1), 1-16. doi: 10.7764/psykhe.29.1.1579

Bourdieu, P. (2014). Las estrategias de la reproducción social. Buenos Aires: Siglo XXI Editores.

Burkett, V. (2013). Coping Capacity. En P. Bobrowsky (Ed.), Encyclopedia of Natural Hazards. Encyclopedia of Earth Sciences Series (pp. 119-129). Dordrecht: Springer. doi: 10.1007/978-1-4020-4399-4

Cámara Chilena de la Construcción (s.f). Déficit habitacional: Un desafío pendiente. Consultado el 28 de julio de 2019. https://www.cchc.cl/2019/deficit-habitacional

Carrero, V., Soriano, R. \& Trinidad, A. (2012). Teoría fundamentada "Grounded Theory": El desarrollo de teoría desde la generalización conceptual. Madrid: CES.

Coller, X. (2005). Estudios de casos. Madrid: CES.

Contreras, Y. \& Beltrán, M. (2015). Reconstruir con capacidad de resiliencia: El casco histórico de la ciudad de Constitución y el sitio del desastre del terremoto y tsunami del 27 de febrero 2010. Revista INVI, 30(83), 79-115. doi: 10.4067/S0718-83582015000100003

Cortés, I. \& Tchernitchin, A. (2018). Metales y metaloides en muestras de polvo depositado en diferentes sectores de Atacama, afectados por los aluviones de marzo 2015". En G. Vargas, S. Pérez \& P. Aldunce (Eds.), Aluviones y resiliencia en atacama: construyendo saberes sobre riesgos y desastres (pp. 181-200). Santiago: Social-Ediciones. doi: $10.34720 / \mathrm{jr} 4 \mathrm{r}-6 \mathrm{j} 31$

Flick, U. (2007). Introducción a la investigación cualitativa. Madrid, España: Ediciones Morata.

Gaillard, J., Cadag, J. \& Rampengan, M. (2019). People's capacities in facing hazards and disasters: an overview. Natural Hazards, 95(3), 863-876. doi: 10.1007/s11069-018-35191

González-Muzzio, C. (2013). El rol del lugar y el capital social en la resiliencia comunitaria posdesastre: Aproximaciones mediante un estudio de caso después del terremoto del 27/F. EURE (Santiago), 39(117), 25-48. doi: 10.4067/S0250-71612013000200002

Günther, M. \& Gutiérrez, R. (2017). La política del ambiente en América Latina. Una aproximación desde el cambio ambiental global. Buenos Aires: CLACSO. doi: 10.2307/j.ctvtxw2j4

Intergovernmental Panel on Climate Change. (2014). Climate change 2014: Impacts, adaptation, and vulnerability. ipcc's fifth anual report. Geneva: IPCC.

Kendra, J., Clay, L. \& Gill, K. (2018). Resilience and Disaster. En H. Rodríguez, W. Donner \& J. Trainor (Eds.), Handbook of disaster research (pp. 87-108). Cham: Springer. doi: 10.1007/978-3-319-63254-4

Lillo, M., Prosser, G., Oróstica, O. \& Pérez, S. (2018). Racionalidades en la gestión del riesgo de desastres: La intervención social en respuesta y recuperación. Revista Sul Americana de Psicología, 6(2), 161-184.

Mabuku, M., Senzanje, A., Mudhara, M., Jewitt, G. \& Mulwafu, W. (2019). Strategies for coping and adapting to flooding and their determinants: A comparative study of cases from Namibia and Zambia. Physics and Chemistry of the Earth, Parts A/B/C. 111, 20-34. doi: 10.1016/j.pce.2018.12.009

Marín, A., Bodin, Ö., Gelcich, S. \& Crona, B. (2015). Social capital in post-disaster recovery trajectories: Insights from a longitudinal study of tsunami-impacted small-scale fisher 
organizations in Chile. Global Environmental Change, 35, 450-462. doi: 10.1016/j.gloenvcha.2015.09.020

Marín, Y., Bedoya, C. \& Cárdenas, G. (2015). Estrategias de adaptación y medios de vida de las familias integrantes de la fundación consejo veredal -fcv-, municipio de Calarcá, Quindío. Luna Azul, (41), 201-239.

Moreno, R. (2019, 12 de abril). Atacama presenta un déficit habitacional de $18 \%$ y es primero a nivel nacional junto a Tarapacá. El Diario de Atacama. https://bit.ly/2QncimO

Organización de las Naciones Unidas para la Alimentación y la Agricultura (s.f). Herramienta para formular, monitorear y evaluar los proyectos de administración de tierras en América Latina. Consultado el 28 de julio de 2019. https://bit.ly/31tdQBR

Rakodi, C. \& Lloyd-Jones, T. (2014). Urban livelihoods: A people-centred approach to reducing poverty. London: Earthscan publications Ltd.

Räsänen, A., Juhola, S., Nygren, A., Käkönen, M., Kallio, M., Monge, A. M. \& Kanninen, M. (2016). Climate change, multiple stressors and human vulnerability: a systematic review. Regional Environmental Change, 16(8), 2291-2302. doi: 10.1007/s10113-016-0974-7

Relaves (s.f). ¿Dónde están los relaves? Consultado el 30 de julio de 2019. https://www.relaves.org/donde_estan.php

Rojas-Páez, L. \& Sandoval-Díaz, J. (2020). Habitando "no lugares": subjetividad y capacidades familiares ante un desastre socionatural en Chile. Íconos. Revista de Ciencias Sociales, 66(24), 59-80. doi: 10.17141/iconos.66.2020.3883

Sandoval, J. (2017). Dimensión subjetiva de la vulnerabilidad ante riesgos y desastres socionaturales: cuatro casos de estudio (tesis de doctorado). Universidad de Chile, Santiago, Chile.

Sandoval-Díaz, J. (2020). Vulnerabilidad-resiliencia ante el proceso de riesgo-desastre: Un análisis desde la ecología política. Polis (Santiago), 19(56), 214-239. doi: 10.32735/s0718-6568/2020-n56-1527

Sandoval Díaz, J. \& Astudillo Pizarro, F. (2019). Comunidades en movimiento ante el cambio climático. ¿Resistentes o resilientes? El caso de Paipote, Chile. Ecología Política. Cuadernos de Debate Internacional, 58, 79-83.

Sandoval-Díaz, J. \& Cuadra-Martínez, D. (2020). Vulnerabilidad social, severidad subjetiva y crecimiento postraumático en grupos afectados por un desastre climatológico. Revista de Psicología, 29(1), 1-15. doi: 10.5354/0719-0581.2020.58002

Sandoval, J., Rojas, L., Villalobos, M., Sandoval, C., Moraga, F., \& Aguirre, N. (2018). De organización vecinal hacia la gestión local del riesgo: diagnóstico de vulnerabilidad y capacidad. Revista INVI, 33(92), 155-180. doi: 10.4067/S0718-83582018000100155

Sandoval, V., \& Sarmiento, J. (2018). Una mirada desde la gobernanza del riesgo y la resiliencia urbana en América Latina y el Caribe: Los asentamientos informales en la Nueva Agenda Urbana. REDER, 2(1), 38-52.

Scoones, I. (2009). Livelihoods perspectives and rural development. The journal of peasant studies, 36(1), 171-196. doi: 10.1080/03066150902820503

Serrat, O. (2017). The sustainable livelihoods approach. En O. Serrat (Ed.) Knowledge solutions: Tools, Methods, and Approaches to Drive Organizational Performance (pp. 21-26). Singapur: Springer. doi: 10.1007/978-981-10-0983-9

Ugarte, A. \& Salgado, M. (2014). Sujetos en emergencia: acciones colectivas de resistencia y enfrentamiento del riesgo ante desastres; el caso de Chaitén, Chile. Revista INVI, 29(80), 143-168. doi: 10.4067/S0718-83582014000100006

UN Office for Disaster Risk Reduction. (2015). Sendai framework for disaster risk reduction 2015-2030. Geneva: UNDRR. 
UN Office for Disaster Risk Reduction. (2019, enero 23). 2018: 60 millones de personas resultaron afectadas por diversos eventos meteorológicos extremos. UNDRR. https://bit.ly/2WMflIN

Wisner, B., Blaikie, P., Cannon, T. \& Davis, I. (2004). At risk. Natural hazards, people's vulnerability and disasters ( $2^{\mathrm{a}} \mathrm{ed}$.). London: Routledge.

Contribución de los autores: a) Concepción y diseño del trabajo; b) Adquisición de datos; c) Análisis e interpretación de datos; d) Redacción del manuscrito; e) revisión crítica del manuscrito.

J.S.D. ha contribuido en a,b,c,d,e; V.K.P. en c,d,e ; S.T.C. en c,d; M.CH.G. en c,d; G.G.B. en e,d; K:A:S en c,d.

Editora científica responsable: Dra. Cecilia Cracco 